\title{
Gene expression analysis of human hepatocellular carcinoma by using full-length cDNA library
}

\author{
Chia-Chu Tsai ${ }^{1}$, Kai-Wen Huang ${ }^{2}$, Hsiao-Fen Chen ${ }^{1}$, Bo-Wen Zhan ${ }^{1}$, Yen-Han Lai ${ }^{1}$, \\ Fa-han Lee ${ }^{1}$, Chung-Yei Lin ${ }^{1}$, Yi-Cheng $\mathrm{Ho}^{1}{ }^{1}$, Yu-Wei Chao ${ }^{1}$, Yih-Ching Su${ }^{1}$, Sen-Wen \\ Jane $^{1}$, Yu-Chi Chen ${ }^{1}$, Chyong-Ing Hsu ${ }^{1}$, Po-Huang $\mathrm{Li}^{2}$, Hey-Chi Hsu ${ }^{3}$, Yutaka Suzuki ${ }^{4}$, \\ Sumio Sugano ${ }^{4}$ \& Jung-Yaw Lin ${ }^{1, *}$ \\ ${ }^{1}$ Institute of Biochemistry and Molecular Biology, College of Medicine, National Taiwan University, Taipei, \\ 100, Taiwan, R.O.C; ${ }^{2}$ Department of Surgery, National Taiwan University Hospital, Taipei, 100, Taiwan, \\ R.O.C; ${ }^{3}$ Department of Pathology and Graduate Institute of Pathology, College of Medicine, National \\ Taiwan University, Taipei, 100, Taiwan, R.O.C; ${ }^{4}$ Laboratory of Genome Structure Analysis, Human Genome \\ Center, Institute of Medical Science, University of Tokyo, Tokyo, Japan
}

(C) 2006 National Science Council, Taipei

Key words: full-length cDNA library, gene expression, HCC, real-time PCR, transcriptome

\section{Summary}

Hepatocellular carcinoma (HCC) is a leading cause of death worldwide. Hepatitis B virus (HBV) or hepatitis $\mathrm{C}$ virus (HCV) infection has been shown to cause hepatic carcinogenesis. A total 58,251 of cDNA clones of full-length cDNA libraries of HBV and HCV-infected HCC and their surrounding non-tumor tissues, respectively, were sequenced and analyzed by blasting against GENEBANK maintained by NCBI. About 180 and 279 of genes were shown an obviously increased and decreased expression patterns between HCC tissue and its adjacent non-tumor tissue. The candidate genes consisted of the genes encoded liver specific metabolism enzymes, secretory functional proteins, proteases and their inhibitors, protein chaperon, cell cycle components, apoptosis-related proteins, transcriptional factors, and DNA binding proteins. Several genes were further investigated by using real-time PCR to confirm the gene expression levels in at least 24 pairs of HCC tissues and adjacent non-tumor tissues. The results showed that genes encoded reticulon 4, RGS-1, antiplasmin, and kallikrein B were down-regulated with the average of 2.8, 8.5, 3.2, and 10.5-fold, respectively. Our results provide crucial candidate genes to develop clinical diagnosis and gene therapy of HCC.

\section{Introduction}

Hepatocellular carcinoma (HCC) is the highly prevalent cancer disease worldwide [1,2]. Hepatic $\mathrm{B}$ virus (HBV) and hepatic $\mathrm{C}$ virus (HCV) have been suggested to be the most important risk factors of hepatocarcinogenesis as well as environmental carcinogens such as aflatoxin B1 and alcoholic

*To whom correspondence should be addressed. Tel.: + 886-223954776; Fax: +886-2-23415334; E-mail: jylin@ha.mc.ntu. edu.tw cirrhosis [3-6]. In recent years, numerous reports have given rise to information on multiple genetic changes such as chromosome aberrations, genetic alternations, and genetic product abnormalities which have been suggested to cause the hepatocarcinogenesis $[2,3,7]$. The genetic changes are determined by using microarray $[8,9]$, comparative genomic hybridization (CGH) [1, 10-12], and serial analysis of gene expression (SAGE) [13]. Despite rapid information gained from these researches, the relationships between molecular mechanism of 
hepatocarcinogenesis and molecular genetics of HCC and its clinical importance remain unclear. By comparative genomic hybridization (CGH) analysis, several chromosomal arms have been demonstrated to be frequent loss of heterozygosity (LOH) such as 1p, 1q, 2q, 4q, 5q, 6q, 8p, 8q, 9p, 9q, 10q, 11p, 13q, 16p, 16q and 17p in HCC while the gain of DNA copy is detected at chromosome 1q, $6 \mathrm{p}, 8 \mathrm{q}$, and $17 \mathrm{q}$ [1]. These indicate that genes located at these chromosomes may be important for carcinogenesis of HCC, probably containing tumor suppressor genes (TSGs) and oncogenes.

In order to investigate the gene expression pattern, the analysis of whole transcriptome provides reliable evidence for the existence by measuring the mRNA molecules expressed in EST library derived from tumor and non-tumor tissues [14]. To address this purpose, the oligo-capping method $[15,16]$ was used to construct the fulllength cDNA library of HCC and non-tumor adjacent liver (NTAL). Total 36,082 and 22,169 clones of cDNA library of HCC and NTAL have been subjected to nucleotide sequencing. $5^{\prime}$-end of each inserts were sequenced with an ABI nucleotide sequencer model 3730 , and the results from paired HCC/liver samples were grouped into 52,629 known cDNAs and 5652 unknown cDNAs $(p$ value $<1 \times 10^{-6}$ ), and further integrated into 3513 and 2692 genes for HCC and NTAL, respectively. The genes were further analyzed their expression differences between HCC and normal liver tissues by comparing the number of cDNA clones.

\section{Materials and methods}

\section{Tissue}

Three HCC tissues which were infected by hepatitis $B$ and hepatitis $C$ virus were obtained the National Taiwan University Hospital. After the tissue slicing from the liver, the tissues were washed with nuclease-free PBS and frozen immediately in liquid nitrogen.

\section{Isolation of total $R N A$ and $m R N A$}

The total RNAs of HCC tissue were purified with standard protocol by using Trizol reagent (Invitrogen), and purified with RNeasy kit (Qiagen) for removing genomic DNA fragment. A poly-d(T) cellulose (Molecular Research Center) was used for isolation of mRNA from the total RNAs according to the standard method. The purified mRNAs were ethanol-precipitated and re-dissolved for further enzyme reaction. The quality of the RNA samples was analyzed by its absorbance ratio of $\mathrm{OD}_{260 / 280}$ which was above 1.8 analyzed by $1 \%$ agarose gel electrophoresis.

\section{Modification of $m R N A$ with oligo-capping method}

The oligo-capping method was carried out as described previously $[15,16]$. The mRNAs with the cap structure was enzymatically modified as in the following three steps: (1) the phosphate of truncated mRNA $5^{\prime}$ ends whose cap structures have been broken down during purification step was hydrolyzed with bacterial alkaline phosphatase (BAP; Takara); (2) tobacco acid pyrophosphatase (TAP; Wako) was used to remove the cap structures of full-length mRNA, leaving the phosphate at the $5^{\prime}$ end; and (3) the $5^{\prime}$ oligo (5'-AGCAUCG AGUCGGCCUUGUUGGCCUACUGG- $3^{\prime}$ ) was ligated to the $5^{\prime}$ end phosphate of BAP-TAP treated mRNA using T4 RNA ligase (Takara). Between each steps, the reaction products were extracted with phenol: chloroform (1:1) to remove the enzyme. The oligo-capped mRNA products were precipitated and re-dissolved for further 1st strand cDNA synthesis.

\section{1st strand cDNA synthesis and $c D N A$ amplification}

The 1st strand cDNA was synthesized by using Superscript II kit (Invitrogen) by combining of the oligo-capped mRNA and $10 \mathrm{pmol}$ of oligo-d(T) adaptor primer (5'-GCGGCTGAAGACGGCC TATGTGGCCTTTTTTTTTTTTTTTTT- $3^{\prime}$ ). The reverse transcription reactions were carried out by incubation at $42{ }^{\circ} \mathrm{C}$ for $18 \mathrm{~h}$. After 1 st strand synthesis, RNA was degraded in $15 \mathrm{mM} \mathrm{NaOH}$ by incubating at $65^{\circ} \mathrm{C}$ for $1 \mathrm{~h}$. The cDNA amplification reaction was carried out by combining $1 \mu \mathrm{g}$ of 1st strand cDNA, 16 pmol of 5' (5'-AGCATCG AGTCGGCCTTGTTG-3') and $3^{\prime}$ ( $5^{\prime}$-GCG GCT GAAGACGGCCTATGT-3') PCR primers with XL PCR kit (Applied Biosystem). The thermocycler condition was 15 cycles at $94{ }^{\circ} \mathrm{C}$ for $1 \mathrm{~min}$, $58^{\circ} \mathrm{C}$ for $1 \mathrm{~min}$, and $72{ }^{\circ} \mathrm{C}$ for $10 \mathrm{~min}$. The amplified cDNA was extracted with phenol:chloroform, and then precipitated with ethanol. 


\section{Size fractionation and library construction}

The cDNA was digested with $S f i$ restriction enzyme (New England Biolabs) by incubating at $37^{\circ} \mathrm{C}$ overnight, and the reaction products were extracted with phenol: chloroform, and then precipitated with ethanol. The SfiI digested products were separated by $1 \%$ agarose gel electrophoresis and the size of products larger than 1500 bp were isolated by using Gel Elution kit (Qiagen) and cloned into DraIII pre-digested pME18S-FL3 vector. The plasmids of cDNA library were electroporated into TOP10 E. coli electro-competent cells using Rio-RAD Gene Pulser under standard conditions [22]. To determine the cloning efficiency, $100 \mu \mathrm{l}$ of the transformation mix were planted on the Low Salt LB-Ampicillin $(10 \mu \mathrm{g} / \mathrm{ml})$ agar plates.

\section{High throughput sequencing of $c D N A$ clones}

The $1 / 30(\mathrm{v} / \mathrm{v})$ of the plasmid DNA was electroporated in to TOP10 E. coli, plated on three $22.2 \mathrm{~cm} \times 22.2 \mathrm{~cm}$ Q-trays (Genetix) and randomly picked the independent clones into 384-wells plates by using Q-Pix robot (Genetix). After the E. coli growing at $37^{\circ} \mathrm{C}$ overnight, colony PCR was carried out by adding $1 \mu \mathrm{l}$ of $E$. coli culture medium, $16 \mu \mathrm{mol}$ of $5^{\prime}\left(5^{\prime}\right.$-TCAGTGGATGTTG CCTTTAC-3') and 3' (5'-TGTGGGAGGTTTTT TCTCTA-3') colony-PCR primers with ExTaq kit (Takara) in the thermocycler conditions of a preheat step at $95^{\circ} \mathrm{C} 5 \mathrm{~min}, 30$ cycles of $95^{\circ} \mathrm{C}$ for $1 \mathrm{~min}, 45^{\circ} \mathrm{C}$ for $1 \mathrm{~min}$, and $72^{\circ} \mathrm{C}$ for $1 \mathrm{~min}$, and final extension step at $72{ }^{\circ} \mathrm{C} 10 \mathrm{~min}$. The colony PCR products were purified with MS-384-PCR plates (Millipore) by using BioMek FX robot (Beckmann). The purified colony PCR products were applied to sequencing reaction by using the sequencing primer (5'-GGATGTTGCCTTTACTTCTA-3') and BigDye 3.0 version kit (Applied Biosystem) in the supplier recommended conditions. The sequencing reaction products were purified with MS-384-SEQ plates (Millipore) and sequenced with an ABI 3700 sequencer.

\section{Computer analysis of $c D N A$ sequences}

The sequencing data were collected and analyzed their sequence similarity against the GenBank non-redundant nucleotide library maintained by the National Center for Biotechnology Information (NCBI) with the BLAST program. Clones which sequences exhibited similarities to database sequences, with BLASTN scores higher than 200, were operationally classified as an identified group. The sequence data were further annotated according to the NCBI UniGene database then grouped and analyzed the gene functions by using homemade grouping program. The cloned sequences that failed to match the non-redundant nucleotide library were compared to EST database for confirming as novel genes.

\section{Real-time PCR analysis}

Total RNA was extracted from $\mathrm{HCC}$ and tumor adjacent normal liver tissues using Trizol reagent (Invitrogen). The total RNA was quantitated, and first-strand cDNA was prepared from total RNA using the SuperScript II cDNA Synthesis method (Invitrogen) with oligo-dT primer. The determination of the transcript levels was performed by quantification assays on the basis of real-time RT-PCR with a iCycler instrument (Biorad) using fluorescenceresonance energy-transfer (FRET) hybridization methodology. The specific primer sets were designed by Beacon designer 4.0 (Premier Biosoft International) and listed as below.

About $100 \mathrm{ng}$ of cDNAs was used to carry out the PCR reaction, and the following PCR conditions: $95{ }^{\circ} \mathrm{C}$ for $15 \mathrm{~min}$ followed by 50 cycles with $95{ }^{\circ} \mathrm{C}$ for $10 \mathrm{~s}$, and $60{ }^{\circ} \mathrm{C}$ for $45 \mathrm{~s}$. All PCR reactions were repeated for each sample in three independent experiments.

\begin{tabular}{lll}
\hline Gene name & Sense primer & Antisense primer \\
\hline RGS1 & CAGAAGGAATGTGCCAGTATGG & CTGCGCCTGGATAACTTTCATG \\
antiplasmin & CGTGGGATCTCCGAGCAGAG & GGGACATGGCAATGCTGGTG \\
Kallikrein B & AAACTCCAGGCTCCTTTGAATTAC & TTTGGATTTCACCTTTCTCCTTCG \\
RTN4 & GGTTGTTGACCTCCTGTACTGG & GAATACTGTCAATGAAAGCAGCAG \\
\hline
\end{tabular}




\section{Results}

The gene expression differences of HCC genes in Tumor $(\mathrm{T})$ and normal tissues $(\mathrm{N})$ were identified by comparing their expression patterns, and total 180 and 279 genes were found to be the significantly up-regulated (Expression Ratio ${ }_{\mathrm{T}}$ / $\mathrm{N}>3.0$ ) and down-regulated (Expression Ratio $_{N} / \mathrm{T}>3.0$ ) genes, respectively (Table 1 ). The genes were further grouped with their cellular functions identified by using GOA term in NCBI (Table 2). A majority of the detoxification protein genes involved in scavenging free radicals, such as glutathione peroxidases, catalase and glutathione S-transferase were highly down-regulated, indicating that the protection ability against oxidative stress was reduced in HCC tissues. The results also showed that most of liver-secreted plasma functional protein genes, such as transferrin, fibrinogen and coagulation factor genes, were significantly down-regulated in HCC tissue, and a numerous of liver-specific metabolic enzymes of xenobiotic genes in which cytochrome P450 and aldehyde dehydrogenase family genes were down-regulated in HCC. These results indicated that down-regulation of these genes may reflect the poor differentiation of hepatocytes and loss of the liver biological functions in HCC tissues.

Moreover, numerous immune-related genes were decreased in HCC tissues, and it may result in reducing the immune-responsive activity against tumor cells. Remarkably, several genes related to protease inhibitors e.g. majority of serine (or cysteine) proteinase inhibitor (SERPIN) family, inter-alpha globulin inhibitors, and cystatin-derived genes, such as histidine-rich glycoprotein (HRGP), $\alpha 2$-HS-glycoprotein (fetuin), and kinin-

Table 1. Analysis of full-length cDNA library.

\begin{tabular}{lll}
\hline & HCC & $\begin{array}{l}\text { Non-tumor } \\
\text { adjacent Liver }\end{array}$ \\
\hline Total clones & 36,082 & 22,169 \\
Total clusters & 3513 & 2692 \\
Up-regulation $^{\mathrm{a}}$ & 180 & \\
Down-regulation $^{\mathrm{a}}$ & 279 & \\
\hline
\end{tabular}

a The up- and down-regulation denoted expression ratio in Tumor/Liver $>3.0$ and expression ratio Liver/Tumor > 3.0, respectively.
Table 2. Groups of up- and down-regulated genes indicated in gene number.

\begin{tabular}{lrc}
\hline Class & $\begin{array}{c}\text { Up- } \\
\text { regulation }\end{array}$ & $\begin{array}{c}\text { Down- } \\
\text { regulation }\end{array}$ \\
\hline Apoptosis & 4 & 1 \\
Cell adhesion & 1 & 1 \\
Cell cycle & 3 & 1 \\
Cytoskeleton & 7 & 7 \\
Degradation & 8 & 7 \\
Detoxification & 2 & 10 \\
DNA binding/gene regulator & 10 & 15 \\
DNA repair & 2 & 1 \\
DNA replication & 1 & 1 \\
Growth factor & 1 & 1 \\
Immune & 5 & 14 \\
Membrane & 5 & 6 \\
Metabolism & 33 & 71 \\
Mitochondria & 4 & 2 \\
Protease & 8 & 2 \\
Protease inhibitor & 2 & 16 \\
Receptor & 7 & 7 \\
Secretion & 2 & 36 \\
Signal transduction & 7 & 18 \\
Stress response & 10 & 3 \\
Transcription/post-transcription & 10 & 7 \\
Translation/post-translation & 9 & 5 \\
Transport & 10 & 28 \\
Tumor association & 7 & 5 \\
Unknown & 14 \\
Total & 279 \\
\hline & & \\
& 70 & 1 \\
\hline
\end{tabular}

ogen, showed the down-regulated expression pattern in HCC tissues compared to those of NTAL tissues. On the contrary, some of the protease genes, such as alanyl aminopeptidase and carboxypeptidase D genes were found to be upregulation in HCC. Taken together, the downregulation of protease inhibitors and up-regulation of protease genes may result in a high potential of tumor invasiveness and metastasis in HCC progression.

In the meanwhile, several genes encoded proteins, which are important in signal transduction, cell cycle regulation, gene expression regulation, were also deregulated in HCC. Stressinduced cherperons, such as heat shock protein 60,70 and 90, have been suggested to be involved in oncogenesis and were found to be up-regulated in HCC. In addition, several genes directly or indirectly involved in signal transduction 
or transcriptional regulation were shown to be up-regulated and down-regulated patterns. In which, RTN4 (reticulon 4), also named ASY/ NOGO, has been suggested to be a tumor suppressor gene [17], and was shown to be down-regulated in tumor tissues, whereas activating transcriptional factor 4 (ATF4) which is response to hypoxic stress and regulating gene expression of CHOP (C/EBP homologous protein) and VEGF (vascular endothelial growth factor) was shown to be overexpressed in tumor tissues.

\section{Identification of putative TSGs and oncogenes}

Based on the previous studies on chromosomal alternation of $\mathrm{HCC}$, highly frequent $\mathrm{LOH}$ and gain of DNA copy have been demonstrated at a numerous chromosomal loci, indicated that putative TSGs and oncogenes could be characterized. By analysis of the full-length cDNA library, 37 genes located in these chromosomal regions showed significant up- or down-regulation in HCC (Table 3). The loss of DNA at 1p36.1 may hit the high-mobility group nucleosomal binding domain 2 which forms specific complexes with nucleosome and is released from mitotic chromosomes during the cell cycle. Besides, the nuclear receptor 0B2 (former SHP1) which represses nuclear hormone receptor-mediated transactivation was found to be down-regulation and located at chromosome 1p36.1. Moreover, chromosome $17 \mathrm{p} 12 \sim 13$ was considered to be frequently loss during hepatocarcinogenesis, and 5 down-regulated genes were within this locus, in which GABA receptor-associated protein is identified as a TSG and has shown reduced expression in breast cancer.

Amplification of chromosome 17q21 contains a growth factor granulin with the ability of promoting cell growth and the G-protein-coupled receptor $\mathrm{C} 5 \mathrm{C}$ gene which may mediate the cellular effects of retinoic acid on the $G$ protein signal transduction cascade. 1q21 is also an important amplified core region on chromosome 1q, two tumor associated genes, e.g. Cisplatin resistance associated gene and CTCL tumor antigen HD-CL-06 was identified up-regulated in present study.

Table 3. Down-regulated genes identified by gene locations within frequent deleted chromosomal arms.

\begin{tabular}{llcc}
\hline Unigene No. & Down-regulation & Fold & Cytoband \\
\hline Hs.181163 & HMGN2: high-mobility group nucleosomal binding domain & 3.0 & $1 \mathrm{p} 36.1$ \\
Hs.427055 & NR0B2: Nuclear receptor subfamily 0, group B, member 2 & 3.0 & $1 \mathrm{p} 36.1$ \\
Hs.75431 & fibrinogen, gamma polypeptide & 4.0 & $1 \mathrm{p} 36.1-\mathrm{p} 34$ \\
Hs.444925 & HMGCL: 3-hydroxymethyl-3-methylglutaryl-Coenzyme & 5.0 & $1 \mathrm{p} 36.1-\mathrm{p} 35$ \\
& A lyase (hydroxymethylglutaricaciduria) & & \\
Hs.4 & alcohol dehydrogenase IB (class I), beta polypeptide & 23.0 & $4 \mathrm{q} 21-\mathrm{q} 23$ \\
Hs.2523 & alcohol dehydrogenase 1C (class I), gamma polypeptide & 18.0 & $4 \mathrm{q} 21-\mathrm{q} 23$ \\
Hs.282984 & DHRS8: Dehydrogenase/reductase (SDR family) member 8 & 5.0 & $4 \mathrm{q} 22.1$ \\
Hs.411826 & ubiquitin-conjugating enzyme E2 & 3.0 & $4 \mathrm{q} 24$ \\
Hs.107 & fibrinogen-like 1 & 7.0 & $8 \mathrm{p} 22-\mathrm{p} 21.3$ \\
Hs.374596 & TPT1: tumor protein, translationally-controlled 1 & 20.5 & $13 \mathrm{q} 12-\mathrm{q} 14$ \\
Hs. 181307 & H3F3A: H3 histone, family 3A & 4.0 & $13 \mathrm{q} 22$ \\
Hs.161640 & TAT: tyrosine aminotransferase & 7.0 & $16 \mathrm{q} 22.1$ \\
Hs.512155 & HPR: haptoglobin-related protein & 3.0 & $16 \mathrm{q} 22.1$ \\
Hs.356190 & UBB: ubiquitin B & 11.0 & $17 \mathrm{p} 12-\mathrm{p} 11.2$ \\
Hs.159509 & SERPINF2: serine (or cysteine) proteinase inhibitor, clade F & 4.0 & $17 \mathrm{p} 13$ \\
& (alpha-2 antiplasmin, pigment epithelium derived factor), member 2 & & $17 \mathrm{p} 13$ \\
Hs.129673 & eukaryotic translation initiation factor 4A, isoform 1 & 3.0 & $17 \mathrm{p} 13.1$ \\
Hs.84359 & GABARAP: GABA(A) receptor-associated protein & 4.0 & $17 \mathrm{p} 13-\mathrm{p} 11$ \\
Hs.437178 & ACADVL: Acyl-Coenzyme A dehydrogenase, very long chain & 6.0 & \\
\hline
\end{tabular}


Reticulon 4
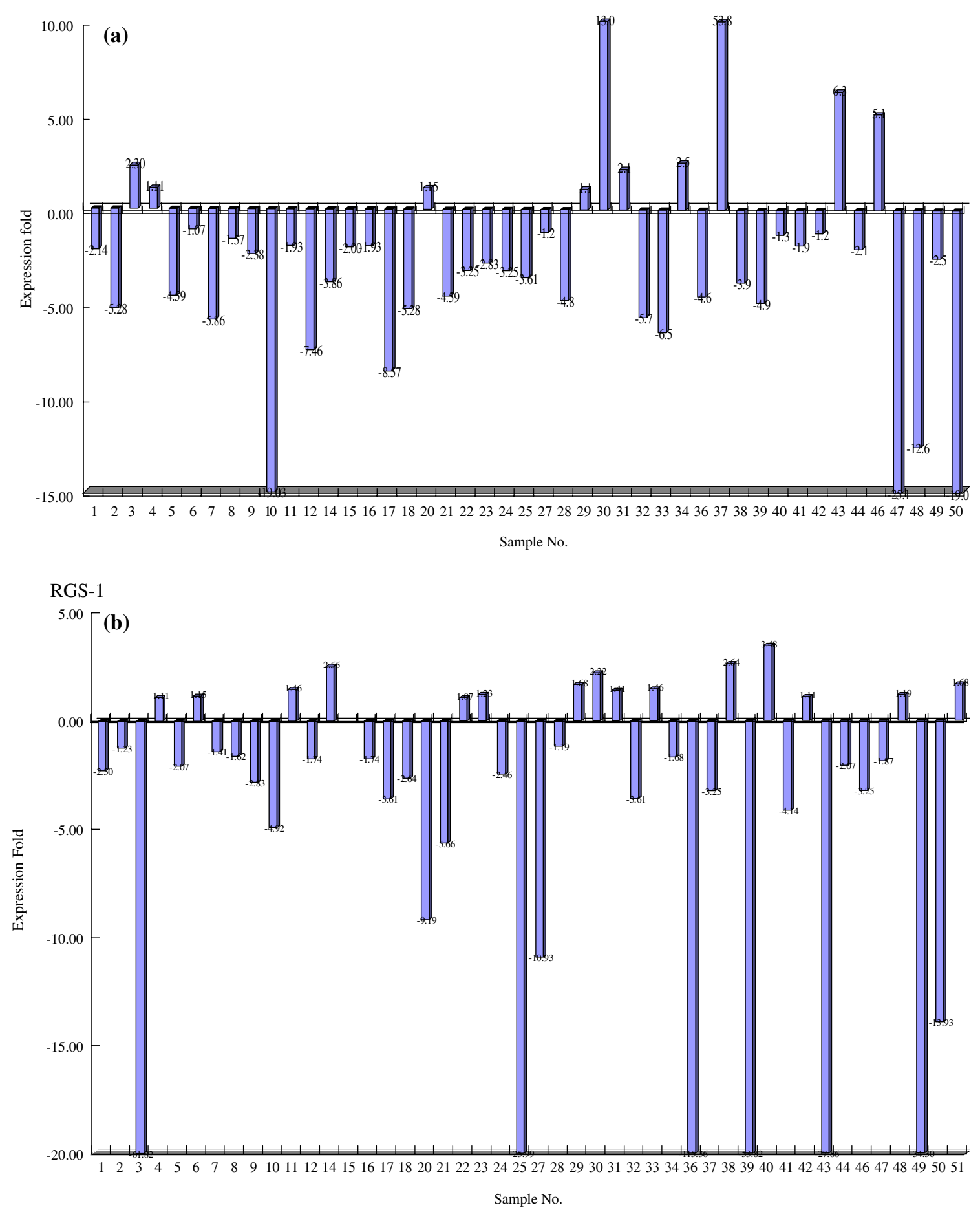

Figure 1. Analysis of gene expression profiles of RTN4, RGS1, antiplasmin and kallikrein B by using real-time PCR. The gene expression patterns for (a) RTN4, (b) RGS1, (c) antiplasmin, and (d) Kallikrein B in HCC samples and their non-tumor adjacent liver samples were determined by real-time PCR. All data were normalized by comparing with those of housekeeping gene GAPDH and are presented as relative fold expression change. A positive value depicts a higher expression level, whereas a negative value showed a lower expression level in tumor relative to the liver samples. All data was the average of at least duplicate of experiments. 


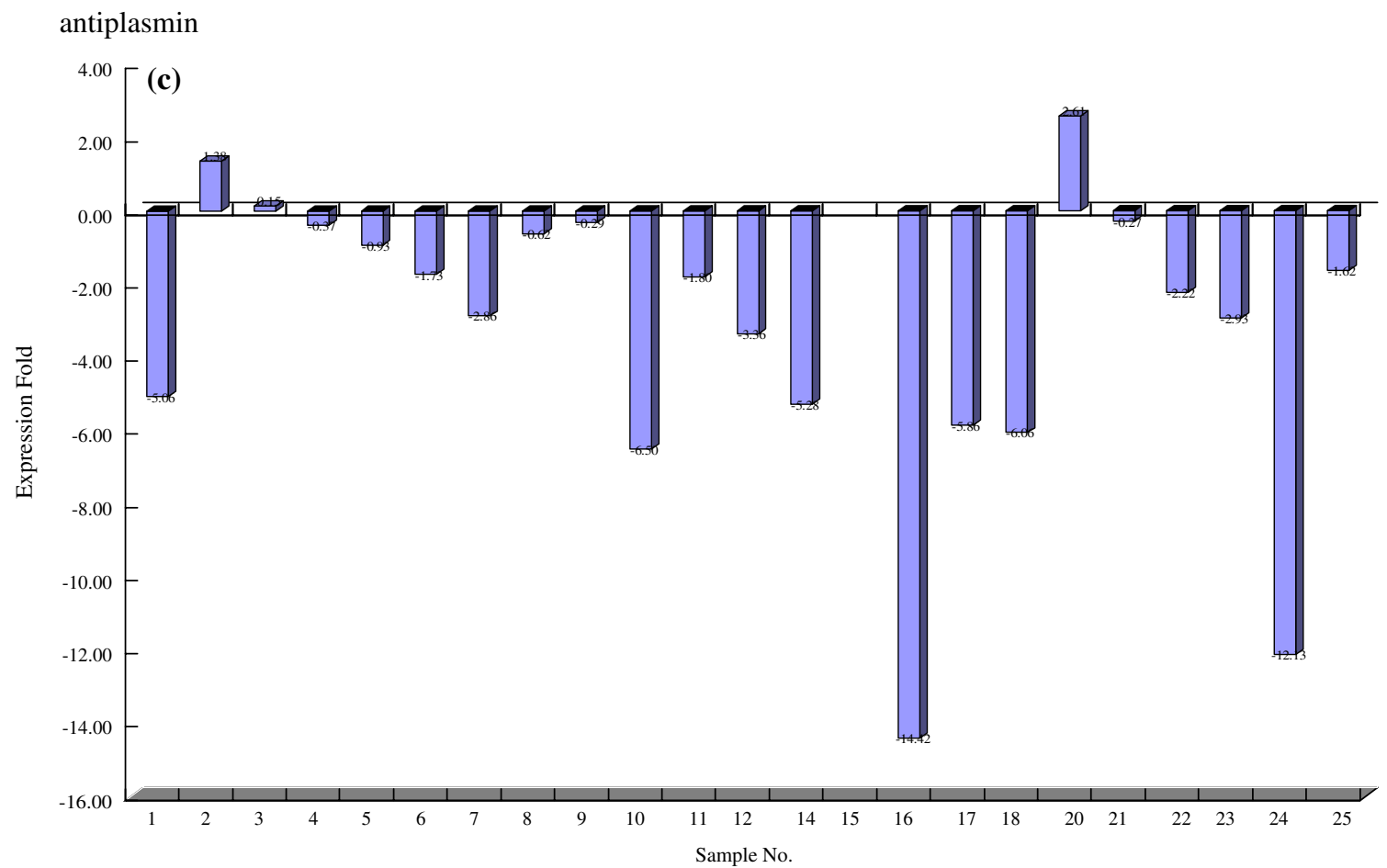

kallikrein B

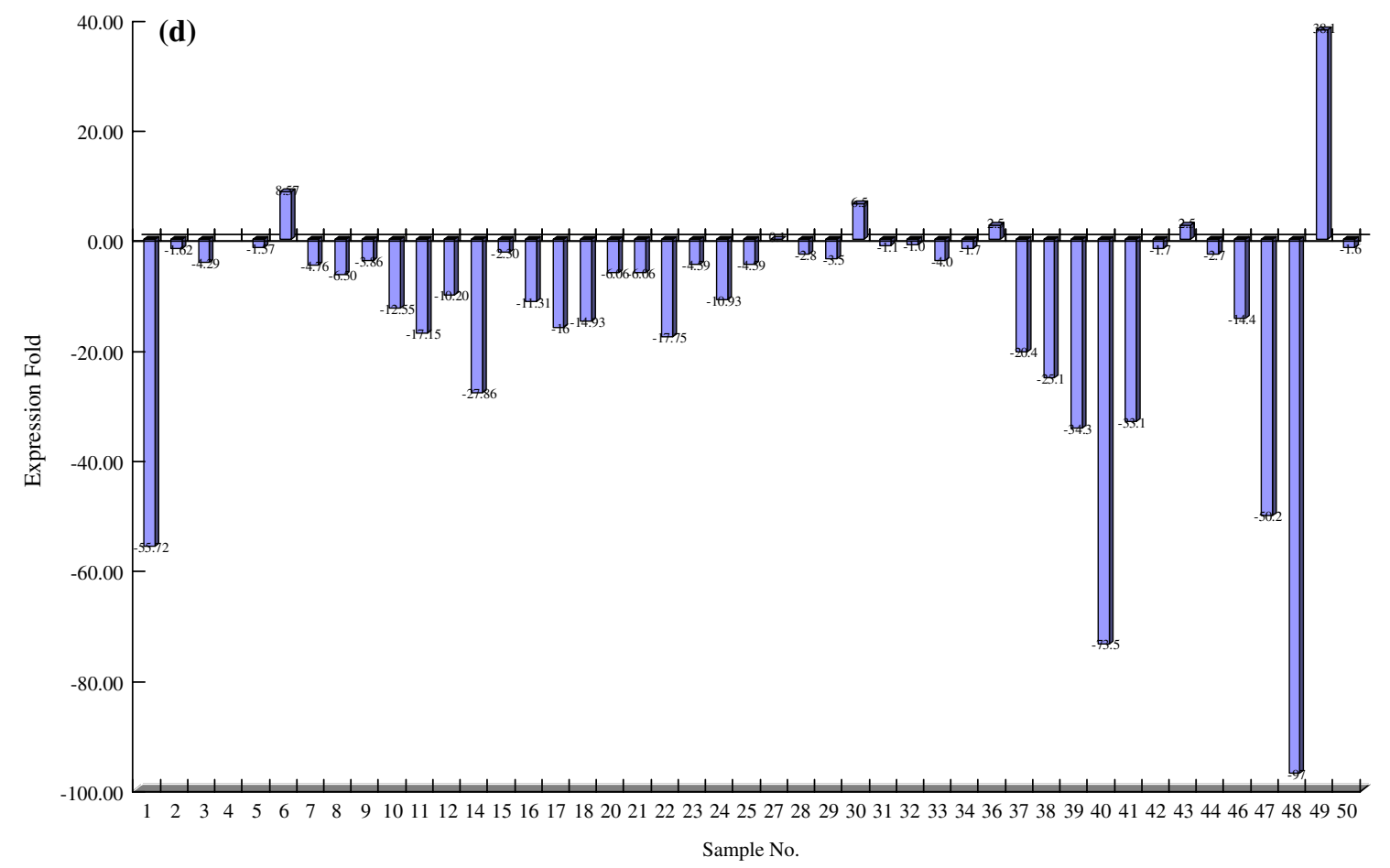

Figure 1. Continued. 


\section{Confirmation of gene expression pattern in HCCs and their paired normal tissues by using Q-PCR}

The deregulation of these candidate genes, i.e. RTN4, antiplasmin, RGS1 (regulator G-protein signaling 1) and kallikrein were confirmed by using Q-PCR in HCC compared to NTAL tissues. Interestingly, some genes whose functions may strongly correlate with cell growth, signal transduction, and liver functions were observed deregulated. In this study, several genes, such as antiplasmin, related to SERPIN were deregulated in HCC samples comparing to the non-tumor adjacent liver samples (Figure 1c). In which, down-regulation of RTN4, a tumor suppressor gene which can cause growth arrest by contact inhibition, has been demonstrated; and the signal transduction component, RGS1, involved in repression of G-protein signaling was decreased in HCCs (Figure 1a, b). The regulation of the signal transduction pathway is important in normal cellular function, and deregulation of signal transduction always plays an important role in tumorigenesis. Moreover, some of the functional proteins secreted by liver e.g. kallikrein B, a majority of coagluation components, showed the down-regulated expression pattern in HCC tissues compared to non-tumor adjacent liver tissues (Figure 1d), and this observation may also suggest these proteins are correlated with poor differentiation of liver.

\section{Discussion}

The full-length cDNA library was analyzed by high-throughput DNA sequencing, and all sequencing results were blasted and grouped into 3513 and 2692 genes in HCC and tumor adjacent normal liver tissues, respectively. The expression patterns were further investigated by comparing the clone numbers of each gene found in the library of HCC tissues with those in tumor adjacent normal liver tissues.

The up- and down-regulated genes were further grouped with their biological functions. The results showed that most of liver-synthesized plasma functional protein genes, such as transferrin, heptoglobin-related protein and coagulation factors, were obviously decrease down-regulated in HCC tissue. These proteins not only play important roles in the well-known biological functions but also have been identified as effectors in cancer biology. For example, kallikrein B is a glycoprotein that participates in the surface-dependent activation of blood coagulation, fibrinolysis. The kallikrein is also involved in activation of tumorassociated pro-urokinase-type plasminogen activator which promotes plasminogen activation and thus the dissolution of the tumor matrix and the basement membrane in prostate cancer [18]. However, down-regulated expression of kallikrein B was found in 31 of $45 \mathrm{HCC}$ tissues, and it may cause by the poor differentiation of liver cancer cells. Besides, a numerous of liver-specific metabolic enzymes of xenobiotics in which cytochrome P450 families were down-regulated in HCC, and down-regulation of these enzymes results in loss of xenobiotic metabolism. Down-regulation of these genes may reflect loss of the liver biological functions in HCC tissues.

During the hepatocarcinogenesis, the downregulation of tumor suppressor gene, RTN4, was investigated in present study. H-rev107 is an ubiquitously expressed in most human tissues and localized in ER membrane. A recent study has suggested that Nogo-B might function as a proapoptotic protein. RTN4 interact with antiapoptotic members of the Bcl-2 family (Bcl-2, Bcl-xL), and Nogo-B and RTN1-C could sequester Bcl-2 and $\mathrm{Bcl}-\mathrm{xL}$ from mitochondria to the $\mathrm{ER}$, thus reducing their antiapoptotic effect [17]. Moreover, the down-regulation of protease inhibitors and upregulation of protease may result in a high potential of tumor invasiveness and metastasis in HCC progression. In which, antiplasmin, also named SERPINF1, has been proofed to inhibit angiogenin-induced endothelial cell invasion and angiogenesis by blocking the angiogenin interaction with its receptor [19]. In present study, $46 \%$ tissue pairs of HCC were shown to be downregulated analyzed by real-time PCR, indicating that this gene may involve in hepatocarcinogenesis.

The results of this study have provided a clue in changes of genetic expression profiles linked to HCC progression. Most of those features may reflect the consequences of genetic aberration such as minimal region deletion, promoter region hypermethylation, and deregulation of transcriptional factors in the hepatocellular carcinogenesis; some of them may be used as clinical diagnostic markers, especially when combined with the 
proteomic analysis. Besides, an overview of the transcriptome status of HCC may enroot the foundation for the further research on the mechanism of clinical and biological significance.

\section{References}

1. Buendia M.A., Genetics of hepatocellular carcinoma. Semin. Cancer Biol. 10: 185-200, 2000.

2. Feitelson M.A., Sun B., Satiroglu Tufan N.L., Liu J., Pan J. and Lian Z., Genetic mechanisms of hepatocarcinogenesis. Oncogene 21: 2593-2604, 2002.

3. Thorgeirsson S.S. and Grisham J.W., Molecular pathogenesis of human hepatocellular carcinoma. Nat. Genet. 31: 339-346, 2002.

4. Bosch F.X., Ribes J. and Borras J., Epidemiology of primary liver cancer. Semin Liver Dis. 19: 271-285, 1999.

5. Fattovich G., Stroffolini T., Zagni I. and Donato F., Hepatocellular carcinoma in cirrhosis: incidence and risk factors. Gastroenterology 127: S35-S50, 2004.

6. Sell S., Heterogeneity and plasticity of hepatocyte lineage cells. Hepatology 33: 738-750, 2001.

7. Tsutsumi T., Suzuki T., Moriya K., Yotsuyanagi H., Shintani Y., Fujie H., Matsuura Y., Kimura S., Koike K. and Miyamura T., Alteration of intrahepatic cytokine expression and AP-1 activation in transgenic mice expressing hepatitis $C$ virus core protein. Virology 304: 415-424, 2002.

8. Chen X., Cheung S.T., So S., Fan S.T., Barry C., Higgins J., Lai K.M., Ji J., Dudoit S., Ng I.O., Van De R.M., Botstein D. and Brown P.O., Gene expression patterns in human liver cancers. Mol. Biol. Cell. 13: 1929-1939, 2002.

9. Zondervan P.E., Wink J., Alers J.C., IJzermans J.N., Schalm S.W., de Man R.A. and van Dekken H., Molecular cytogenetic evaluation of virus-associated and non-viral hepatocellular carcinoma: analysis of 26 carcinomas and 12 concurrent dysplasias. J. Pathol. 192(2): 207-15, 2000 Oct.
10. Niketeghad F., Decker H.J., Caselmann W.H., Lund P., Geissler F., Dienes H.P. and Schirmacher P., Frequent genomic imbalances suggest commonly altered tumour genes in human hepatocarcinogenesis. Br. J. Cancer 85: 697-704, 2001.

11. Raidl M., Pirker C., Schulte-Hermann R., Aubele M., Kandioler-Eckersberger D., Wrba F., Micksche M., Berger W. and Grasl-Kraupp B., Multiple chromosomal abnormalities in human liver (pre)neoplasia. J. Hepatol. 40: 60-8, 2004.

12. Levine A.J., Momand J. and Finlay C.A., The p53 tumour suppressor gene. Nature 351: 453-456, 1991.

13. Thomas M., Kalita A., Labrecque S., Pim D., Banks L. and Matlashewski G., Two polymorphic variants of wild-type p53 differ biochemically and biologically. Mol. Cell. Biol. 19: 1092-1100, 1999.

14. Storey A., Thomas M., Kalita A., Harwood C., Gardiol D., Mantovani F., Breuer J., Leigh I.M., Matlashewski G. and Banks L., Role of a p53 polymorphism in the development of human papillomavirus-associated cancer. Nature 393: 229-234, 1998.

15. Suzuki Y. and Sugano S., Construction of full-lengthenriched cDNA libraries. The oligo-capping method. Methods Mol. Biol. 175: 143-153, 2001.

16. Suzuki Y., Yoshitomo-Nakagawa K., Maruyama K., Suyama S. and Sugano A., Construction and characterization of a full length-enriched and a $5^{\prime}$-end-enriched cDNA library. Gene 200: 149-156, 1997.

17. Watari A. and Yutsudo M., Multi-functional gene ASY Nogo/RTN-X/RTN4: apoptosis, tumor suppression, and inhibition of neuronal regeneration. Apoptosis 8: 5-9, 2003.

18. Goretzki L., Schmitt M., Mann K., Calvete J., Chucholowski N., Kramer M., Gunzler W.A., Janicke F. and Graeff H., Effective activation of the proenzyme form of the urokinase-type plasminogen activator (pro-uPA) by the cysteine protease cathepsin L. FEBS Lett. 297: 112-118, 1992.

19. Gho Y.S., Yoon W.H. and Chae C.B., Antiplasmin activity of a peptide that binds to the receptor-binding site of angiogenin. J. Biol. Chem. 277: 9690-9694, 2002. 